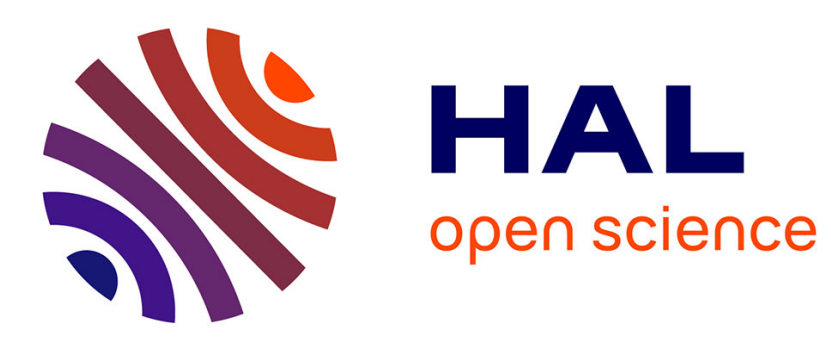

\title{
Épargne de précaution et chômage: une évaluation quantitative de l'auto-assurance
}

Yann Algan, Arnaud Chéron, Jean-Olivier Hairault, François Langot

\section{To cite this version:}

Yann Algan, Arnaud Chéron, Jean-Olivier Hairault, François Langot. Épargne de précaution et chômage: une évaluation quantitative de l'auto-assurance. Annales d'Economie et de Statistique, 2004, 74, pp.105-130. hal-01038116

\section{HAL Id: hal-01038116 \\ https://hal-sciencespo.archives-ouvertes.fr/hal-01038116}

Submitted on 23 Jul 2014

HAL is a multi-disciplinary open access archive for the deposit and dissemination of scientific research documents, whether they are published or not. The documents may come from teaching and research institutions in France or abroad, or from public or private research centers.
L'archive ouverte pluridisciplinaire HAL, est destinée au dépôt et à la diffusion de documents scientifiques de niveau recherche, publiés ou non, émanant des établissements d'enseignement et de recherche français ou étrangers, des laboratoires publics ou privés. 


\title{
Épargne de précaution et chômage : une évaluation quantitative de l'auto-assurance
}

\author{
Yann ALGAN *, Arnaud CHÉRON **, \\ Jean-Olivier HAIRAULT ${ }^{\star \star *}$, François LANGOT **
}

\begin{abstract}
RÉSUMÉ. - L'ambition de ce travail est d'évaluer quantitativement la capacité de l'épargne de précaution à couvrir le risque de chômage dans le cadre d'une économie caractérisée par des risques idiosyncratiques sur le marché du travail. II apparaît que l'épargne de précaution permet de réduire considérablement le coût associé au risque de chômage. Elle offre ainsi un niveau d'assurance relativement comparable au système d'allocation-chômage existant. Cependant, ces résultats découlent de la comparaison d'état stationnaire. II s'avère que le coût de la transition inhérent à l'accumulation d'une épargne de précaution est relativement conséquent. Ensuite, si l'épargne de précaution fournit une assurance efficace en moyenne, elle pénalise les chômeurs de longue durée et augmente ainsi les inégalités de position. Toutefois, elle tend à réduire les inégalités de perspectives, les agents en emplois devant se constituer une épargne de précaution en vue d'épisodes potentiels de chômage.
\end{abstract}

\section{Precautionary Savings and Unemployment: A Preliminary Quantitative Investigation}

ABSTRACT. - The potential welfare benefits of precautionary saving is studied using a quantitative dynamic general equilibrium model. To provide a role for precautionary saving, agents face exogeneous idiosyncratic employment shocks and are unable to borrow or insure themselves through private markets. It appears that precautionary saving allows to decrease the cost of labor market uncertainty to a level comparable with the observed unemployment insurance. However, taking into account the transitory path on which households must provide a huge effort of savings alters this conclusion. A rawlsian criterion leads to be more skeptical about the precautionary saving too.

\footnotetext{
* EUREQua (Université de Paris I).

** GAINS (Université du Maine) \& Cepremap.

*** EUREQua (Université de Paris I) \& Cepremap.

Les auteurs remercie Frank PORTIER pour ses remarques et suggestions sur des versions antérieures de cet article. Nous sommes naturellement seuls responsables des insuffisances qui y demeurent.
} 


\section{Introduction}

Les ménages, confrontés potentiellement au risque de se retrouver sans emploi, ont la possibilité de se constituer un capital dans lequel ils puiseront dans les mauvais jours. On parle traditionnellement d'épargne de précaution définie comme le supplément d'épargne généré par la présence d'incertitude. Certains travaux sur la consommation ont permis de montrer l'importance empirique de ce phénomène (voir, par exemple, ZELDES [1989] et BonNET et DuboIs [1995]). Alors qu'il était traditionnel de fonder théoriquement cette épargne de précaution sur un comportement de prudence liée à la spécification des préférences (voir LELAND [1968] ou KIMBALL [1990]), il apparaît plus pertinent, depuis les travaux précurseurs de AIYAGARI [1994] et de HugGETT [1997], de baser ce comportement sur l'existence de contraintes de liquidité.

Faut-il pour autant considérer l'épargne individuelle comme un moyen efficace de s'assurer contre les aléas liés au marché du travail? Dans quelle mesure cette auto-assurance peut-elle constituer un substitut au système public d'allocation-chômage ?

L'ambition de ce travail est d'évaluer quantitativement la capacité de l'épargne de précaution à assurer les agents contre le risque de chômage dans le cadre d'une économie caractérisée par une hétérogénéité dans la détention d'actifs financiers. Depuis les travaux de AiYAGARI [1994] et de HugGETT [1997], il est possible de traiter de l'hétérogénéité des agents et des mécanismes assurantiels en prenant en compte explicitement l'accumulation d'actifs financiers. Dans un univers incertain et en présence de contraintes de liquidités, les agents se constituent un patrimoine dans lequel ils puisent les ressources nécessaires au lissage de leur consommation lors des périodes de chômage. En fonction de l'ampleur perçue du risque associé au marché du travail, les agents sont alors plus ou moins précautionneux.

Ces comportements d'auto-assurance rejaillissent naturellement sur le problème de l'allocation-chômage optimale. L'évaluation des gains de la mise en place d'un système d'allocation-chômage est en effet indissociables des gains de l'auto-assurance. Le montant de l'épargne de précaution est lié aux risques en termes de revenus sur le marché du travail qui dépendent de l'allocation-chômage, tandis que les coûts associés à une baisse de l'allocation-chômage dépendent de la possibilité de s'auto-assurer. Cependant, paradoxalement, les travaux sur l'assurance-chômage ne prennent traditionnellement pas en compte cette auto-assurance permise par les marchés financiers, ce qui risque de biaiser les recommandations de politique économique. Naturellement, cette limite peut s'expliquer pour des raisons techniques liées à la difficulté de traiter des modèles dynamiques en présence d'hétérogénéité. Certaines études sur l'optimalité du système d'allocationchômage (voir par exemple HANSEN et IMROHOROGLU [1992], ACEMOGLu et Shimer [1999], JosePH et WeITZENBLum [2000]) s'inscrivent cependant dorénavant dans ce cadre dynamique.

Notre objectif dans ce papier est plus modeste qu'une réflexion en profondeur et véritablement appliquée sur le système d'assurance chômage optimal dans une économie comme la France. Notre propos est de révéler le potentiel d'assurance que recèle l'épargne de précaution, et donc de montrer l'impor- 
tance à considérer cet instrument lors de l'évaluation des systèmes de protection sociale dans des travaux plus ambitieux. Est-il comparable avec le système actuel d'allocation-chômage ? Nous comparerons ces deux formes d'assurance à l'aune de deux situations hypothétiques et opposées. La première correspond à la situation de mutualisation complète des risques, où les agents sont parfaitement assurés contre le risque de chômage : elle permet, par opposition, de calculer le coût de l'absence d'assurance associé au risque de chomage dans une économie donnée. La seconde renvoie à une économie où il n'existerait aucune possibilité de s'assurer contre les risques de chômage, les agents prenant de plein fouet les fluctuations de leur revenu qui se répercutent alors totalement sur les variations de leur consommation. Ces deux situations de référence permettent de comparer les capacités assurancielles de l'épargne de précaution relativement à celles du système d'allocationchômage.

Il apparaît que l'épargne de précaution permet de réduire considérablement le coût du risque de chômage. Elle offre ainsi un niveau d'assurance relativement comparable au système d'allocation-chômage. Cependant, cette première conclusion doit être fortement nuancée. D'abord, elle découle de la comparaison d'états stationnaires. Il s'avère que le coût de la transition inhérent à l'accumulation d'une épargne de précaution est relativement conséquent pour la génération qui supporte la suppression d'un système d'assurance publique. Enfin, si l'épargne de précaution fournit une assurance efficace en moyenne, elle ne permet pas au plus pauvre, un chômeur de long terme sans patrimoine, de s'assurer, ce qui conduit à l'aune de ce critère rawlsien à créer plus de différences entre auto-assurance et assurance publique. De façon générale, l'auto-assurance pénalise les travailleurs qui se retrouvent dans une situation anormalement (par rapport à la durée anticipée) longue de chômage. Cette forme privée d'assurance tend ainsi à augmenter les inégalités de position dans l'économie. Toutefois, il faut souligner que l'auto-assurance tend à réduire le différentiel de bien-être espéré entre chômeur et travailleur, réduisant ainsi ce que l'on peut appeler les inégalités de perspectives (ou des possibles). Ce résultat n'est pas contradictoire avec celui obtenu avec un critère rawlsien car dans ce dernier cas on raisonne sur une situation qui ne correspond pas à l'espérance d'un chômeur, mais sur l'histoire très particulière d'un individu atypique.

L'utilisation d'un modèle très stylisé ne permet pas de donner à nos évaluations un caractère opératoire. Nous supposons que l'unique source d'incertitude dans l'économie est la situation sur le marché du travail : être employé, ce qui permet d'obtenir un salaire, ou être chômeur, ce qui n'autorise qu'une activité domestique. Ces différents états sur le marché du travail sont auto-corrélés. Toutefois, ce travail préliminaire montre qu'analyser de façon jointe chômage et accumulation peut être une approche non seulement féconde, mais aussi nécessaire dans un objectif de réflexion sur les mécanismes d'assurance optimaux. Non seulement l'épargne de précaution fournit une assurance, mais les décisions d'entrée et de sortie du chômage peuvent dépendre du niveau de la richesse des agents, ce qui peut encore renforcer l'interdépendance entre ces deux phénomènes (voir Algan, ChÉrON, HAIRAULT et LANGOT [2002]).

Dans une première section, nous présentons un modèle simple permettant de lier les décisions d'accumulation au risque de chômage. Dans une 
deuxième section, nous présentons trois systèmes alternatifs à l'auto-assurance permise par les marchés financiers. Après avoir présenté l'étalonnage et la méthode de résolution du modèle avec accumulation, nous évaluons dans une quatrième section l'épargne de précaution en tant que mécanisme d'assurance au regard d'un système d'allocation-chômage. Finalement, dans une dernière section, nous présentons les limites de l'auto-assurance en levant certaines hypothèses.

\section{Une économie avec épargne de précaution et risque de chômage}

Considérons une économie marquée par un risque sur le marché du travail. Il existe des probabilités non nulles de sortir de l'emploi et de rester au chômage. Dans ce dernier cas, le revenu est tiré d'une activité de production domestique. Pour simplifier, chaque agent fournit la même quantité de travail qu'il soit employé ou qu'il se consacre à une activité domestique. Naturellement il serait possible d'introduire plus d'hétérogénéité dans notre économie, en introduisant des classes supplémentaires de revenus salariaux (voir par exemple HAIRAULT et LANGOT [2002]). Cependant, nous privilégions ici une structure simplifiée qui est suffisante pour mettre en évidence les propriétés de l'épargne de précaution.

\subsection{Risques spécifiques de revenu}

A chaque période de sa vie active, un individu fait face à un risque spécifique sur le marché du travail. Ce risque se traduit par la possibilité de connaître des épisodes de chômage. Ainsi, au cours de son cycle de vie, l'individu est soit employé $(e)$, soit chômeur $(u)$. Les transitions entre ces deux états sont modélisées par le processus de MARKOV suivant :

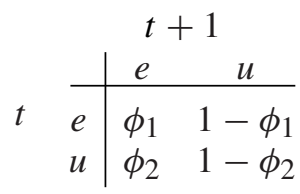

Soit $s$ la variable aléatoire donnant le statut d'un individu sur le marché du travail, on peut alors noter les probabilités de transition entre ces différents états comme les probabilités conditionnelles de la matrice définie ci-dessus :

$$
\pi\left(s^{\prime} \mid s\right)=\operatorname{Pr}\left\{s_{t+1}=s^{\prime} \mid s_{t}=s\right\}
$$

où $s, s^{\prime} \in \mathcal{S}=\{e, u\}$. 
Lorsqu'il est employé, l'individu consacre son temps disponible au travail et reçoit en contre-partie une rémunération. Lorsqu'il est chômeur, l'individu consacre tout son temps à des activités hors-marché (« production domestique »). Cette activité est supposée avoir une efficacité moins importante que l'activité de production dans le secteur marchand. Les revenus sont alors définis par :

$$
w v(s)=\left\{\begin{array}{ccc}
w & \text { si } & s=e \\
\theta w & \text { si } & s=u
\end{array}\right.
$$

où $w$ est le taux de salaire et $v(s)$ est une variable indicatrice permettant de donner le gain net du ménage en fonction de sa situation : elle vaut 1 dans le cas d'un employé et $\theta<1$ dans le cas d'un chômeur, mesurant ainsi l'écart d'efficacité de la production domestique par rapport à la production marchande.

\subsection{Préférences}

Les préférences des agents sont résumées par la fonction d'utilité suivante

$$
\sum_{t=0}^{\infty} \beta^{t}\left\{\sum_{s_{t}} \pi\left(s_{t} \mid s_{t-1}\right) u\left(c_{t}\right)\right\}
$$

où le facteur d'escompte psychologique vérifie $\beta \in] 0,1\left[\right.$, la consommation $c_{t}$ est strictement positive. Le flux instantané d'utilité $u$ est une fonction strictement concave de type CRRA :

$$
u(c)=\frac{c^{1-\sigma}-1}{1-\sigma}
$$

avec $\sigma$ le coefficient d'aversion relative pour le risque.

\subsection{Auto-assurance et accumulation}

Dans cette section, nous présentons les choix individuels d'épargne permettant aux agents de s'auto-assurer contre les risques de revenus. Les ménages ont en effet accès à un actif financier, dont le rendement est certain, ne permettant pas de s'assurer parfaitement contre les risques individuels de revenu. De plus, on suppose que les ménages subissent une contrainte de liquidités (contrainte de non-endettement).

\subsubsection{Les règles de décisions des ménages}

Le vecteur des variables d'état pour le ménage est le vecteur $(k, s)$ où $k$ représente le stock d'actifs financiers de début de période et $s$ la réalisation spécifique à l'agent des événements idiosyncratiques. Le programme résolu par un ménage est le suivant, pour $k_{0}$ et $s_{0}$ donnés : 


$$
v(k, s)=\max _{c \geqslant 0}\left(u(c)+\beta\left\{\sum_{s^{\prime}} \pi\left(s^{\prime} \mid s\right) v\left(k^{\prime}, s^{\prime}\right)\right\}\right)
$$

sous les contraintes

$$
\begin{aligned}
c+k^{\prime} & =(1+r) k+w v(s) \\
k^{\prime} \geqslant 0 &
\end{aligned}
$$

où $v$ représente la fonction-valeur du ménage et $r$ le taux d'intérêt.

\subsubsection{Définition des distributions stationnaires}

Pour un vecteur donné de prix $(r, w)$, les distributions stationnaires de cette économie sont obtenues après avoir déterminé l'ensemble des règles de décision du ménage $\left\{c(k, s), k^{\prime}(k, s)\right\}$, les fonctions-valeur du ménage $v(k, s)$. La distribution stationnaire de probabilité $\lambda(k, s)$, définissant la probabilité inconditionnelle d'avoir un stocks d'actif $k$ dans l'etat $s$ peut alors être calculée. L'état stationnaire vérifie :

(i) Les règles de décision $k^{\prime}=g(k, s)$ et $c=f(k, s)$ sont solutions du programme de maximisation des agents.

(ii) La distribution de probabilité $\lambda(k, s)$ est une distribution stationnaire associée à $\left\{g(k, s), \pi\left(s^{\prime} \mid s\right)\right\}$ vérifiant :

$$
\lambda\left(k^{\prime}, s^{\prime}\right)=\sum_{s} \sum_{k: k^{\prime}=g(k, s)} \lambda(k, s) \pi\left(s^{\prime} \mid s\right)
$$

(iii) L'offre agrégée de capital est alors définie par

$$
K=\sum_{k, s} \lambda(k, s) g(k, s)
$$

Cette offre de capital $K$ donne ici une mesure de l'épargne de précaution, définie comme le supplément d'épargne que se constitue un agent lorsqu'il passe d'une situation certaine à une situation risquée. En effet, dans le cas d'un équilibre partiel ( $r$ exogène), si, comme nous le supposons ${ }^{1}$ $r \in\left[0,(1 / \beta)-1\left[\right.\right.$ et $k_{0}=0$, les choix optimaux des agents en univers certain conduisent à un équilibre sans capital. L'introduction d'un risque de revenu, si la contrainte d'endettement est tel que $k^{\prime} \geqslant 0$, génère alors une offre de capital croissante avec le taux d'intérêt.

L'économie qui vient d'être décrite correspond au cas où seuls les marchés financiers fournissent une assurance contre les risques de revenu. Il s'agit d'une auto-assurance qui passe par des transferts intertemporels. Par la suite, les résultats relatifs à cette situation seront notés $M F$. Nous les comparerons avec trois situations : l'une correspondant à une économie avec assurance parfaite, une deuxième dans laquelle il existe une assurance publique incomplète, et enfin une troisième où aucun instrument ne permet de lisser la consommation.

1. Il aurait été possible de raisonner en écart à une trajectoire décroissante d'épargne si $k_{0}>0$; les résultats quantitatifs ne seraient pas modifiés. 


\section{Trois économies alternatives}

Nous envisageons trois types d'allocation où les risques de chômage sont identiques à ceux présentés dans la section précédente, mais où les possibilités assuriancielles, lorsqu'elles existent, sont basées sur des transferts inter-personnels.

\subsection{Mutualisation complète}

Notre objectif est d'étudier l'efficacité assurancielle de l'épargne de précaution. Cependant, afin d'évaluer les gains ou les pertes en bien-être de différents mécanismes d'assurance contre le risque de chômage, il est utile de se donner une référence : l'allocation optimale, i.e. l'allocation choisie par un planificateur. Ainsi, l'évaluation des mécanismes d'assurance se fera toujours en référence à ce monde idéal où le planificateur mutualise parfaitement les risques associés au marché du travail . Le problème d'allocation optimale est défini par :

$$
\begin{aligned}
& \max \sum_{t=0}^{\infty} \beta^{t}\left[N_{t} u\left(c_{t}^{n}\right)+\left(1-N_{t}\right) u\left(c_{t}^{u}\right)\right] \\
& \text { s.c. }\left\{\begin{aligned}
N_{t} c_{t}^{n}+\left(1-N_{t}\right) c_{t}^{u} & \leqslant N_{t} w_{t}+\left(1-N_{t}\right) \theta w_{t} \\
N_{t} & \leqslant \frac{N}{}
\end{aligned}\right.
\end{aligned}
$$

où $N_{t}$ est le taux d'emploi, $c_{t}^{n}$ et $c_{t}^{u}$ les consommations des employés et des chômeurs, et $w_{t}$ et $\theta w_{t}$ les productions des employés et des chômeurs. De plus, $\bar{N}$ représente la limite de la valeur admissible pour $N_{t}$; elle est donnée par la matrice $\pi$ gouvernant les changements d'état exogènes sur le marché du travail et correspond au niveau inconditionnel de l'emploi. Comme dans ce problème il n'y a plus d'incertitude, il n'y a pas d'épargne ${ }^{2}$. Le problème du planificateur est alors une suite de problèmes statiques ayant comme solution :

$$
\begin{aligned}
& c_{t} \equiv c_{t}^{n}=c_{t}^{u} \\
& c_{t}=\bar{N} w_{t}+(1-\bar{N}) \theta w_{t}
\end{aligned}
$$

Cette allocation $\left(c_{t}^{n}=c_{t}^{u}\right)$ correspond à celle que l'on obtiendrait dans une économie de marché si l'assurance permettait, contre une cotisation de

2. On peut montrer que si le planificateur peut épargner dans les mêmes conditions que les agents privés $\left(k^{\prime} \geqslant 0\right)$, alors, pour $k_{0}=0$, on a nécessairement $k_{t}=0 \forall t$. L'allocation optimale est alors identique à celle du problème sans épargne présentée dans le texte. 
$\tau w_{t}$ lorsque l'agent est employé, de toucher une indemnité de $B_{t}=(1-\theta-\tau) w_{t}$, en cas de chômage ${ }^{3}$.

Si le marché de l'assurance est concurrentiel, la condition de libre entrée donne la prime d'équilibre $\tau=(1-\theta)(1-\bar{N})$. Ainsi, à l'équilibre de marché, on a une indemnité chômage égale à $(1-\theta) w_{t} \bar{N}$. Cette allocation où les risques associés au marché du travail sont parfaitement mutualisés sera notée $M C$, pour « marchés complets ».

\subsection{Allocation-chômage}

Nous considérons maintenant le cas d'une allocation-chômage par laquelle l'État assure partiellement les agents contre le risque de chômage en redistribuant des employés vers les chômeurs. Afin d'identifier les vertus respectives de l'auto-assurance et de cette assurance publique, on suppose que, dans ce dernier cas, les agents n'ont pas accès aux marchés financiers.

Ainsi, le gouvernement taxe les revenus salariaux des ménages afin d'effectuer des transferts vers les chômeurs. En plus de sa production domestique, le chômeur touche une indemnité. Les revenus sont alors définis par :

$$
w v(s)=\left\{\begin{array}{lll}
(1-\tau) w & \text { si } & s=e \\
(\theta+b) w & \text { si } & s=u
\end{array}\right.
$$

On impose $1-\tau>\theta+b . b<1$ est le ratio de remplacement. L'utilité inter-temporelle d'un agent est alors donnée par :

$$
v(s)=u(w v(s))+\beta\left\{\sum_{s^{\prime}} \pi\left(s^{\prime} \mid s\right) v\left(s^{\prime}\right)\right\}
$$

L'équilibre de la contrainte budgétaire du gouvernement est assuré par l'ajustement du taux de cotisation sur le salaire, $\tau$. L'indemnité-chômage est supposée inconditionnelle : indépendante du nombre de périodes passées au chômage (non-dégressive), et il n'y a pas de durée limite pour l'indemnisation. Une étude plus appliquée sur les vertus et limites de l'allocation-chômage devrait naturellement prendre en compte une certaine dégressivité jusqu'à une non-élligibilité. En outre, nous ne prenons pas en compte les distorsions sur l'offre de travail induites par cette taxe. Les résultats relatifs à cette économie seront notés $A C$.

La troisième situation correspondant à l'absence totale d'assurance d'allocation-chômage, s'obtient simplement en posant $b=0$ dans le cas précédent de l'allocation-chômage. Les résultats relatifs à cette configuration seront notés 0 .

3. Cette indemnité $B_{t}$ résulte de l'équivalence des consommations des employés et des chômeurs, définies par les conditions suivantes :

$$
\begin{aligned}
c_{t}^{n} & =(1-\tau) w_{t} \\
c_{t}^{u} & =\theta w_{t}+B_{t}
\end{aligned}
$$




\section{Etalonnage et méthode de résolution}

Afin d'évaluer quantitativement les vertus relatives des différents types d'assurance, il est nécessaire au préalable d'étalonner les modèles. De plus, le modèle intertemporel avec risques idiosyncrasiques sur le marché du travail et accummulation ne pouvant pas être résolu analytiquement, la méthode de résolution numérique utilisée sera également présentée dans ce cas.

\subsection{L'étalonnage}

Les paramètres structurels sont en quasi-totalité commun aux différentes économies considérées (Tableau 1). L'aversion pour le risque est fixée à 2 et le facteur de préférence pour le présent à 0,985 , ce qui correspond à un taux d'escompte psychologique de $1,523 \%$. Ces valeurs sont traditionnellement retenues pour un étalonnage trimestriel (COOLEY [1995]). La probabilité de sortie du chômage est fixée 4 à $31 \%$, ce qui implique une probabilité de sortie de l'emploi égale à 3,45 \% afin de se caler sur un taux de chômage de $10 \%$. Nous considérons un taux d'intérêt réel donné de référence égal à $0,5 \%,{ }^{5}$ un taux de dépréciation égal à $3 \%$, et une part du capital dans le produit égale à $36 \%$. Nous considérons que le travail domestique permet d'atteindre un revenu équivalent à $15 \%$ du salaire ${ }^{6}$. Le salaire est déterminé, pour une fonction de production macroéconomique de type $A K^{\alpha} L^{1-\alpha}$, par la frontière des prix des facteurs $(F P F)$ :

$$
w=(1-\alpha) A\left(\frac{r+\delta}{\alpha A}\right)^{\frac{\alpha}{\alpha-1}}
$$

$A$ est normalisé à un et le taux de salaire est déduit de la $F P F$ étant donnée la calibration de $r$. Cette procédure est cohérente avec celle utilisée lors du calcul de l'équilibre général : le taux d'intérêt et le salaire sont alors endogènes et liés par la $F P F$, la valeur du paramètre d'échelle de la fonction de production restant fixé à un.

\section{TABLEAU 1}

\section{Etalonnage des paramètres structurels}

\begin{tabular}{|ccccccccc|}
\hline$\sigma$ & $\beta$ & $r$ & $\delta$ & $\alpha$ & $A$ & $\theta$ & $\phi_{1}$ & $\phi_{2}$ \\
\hline 2 & 0,985 & 0,05 & 0,03 & 0,36 & 1 & 0,15 & 0,9655 & 0,31 \\
\hline
\end{tabular}

4. Voir Algan et Terracol [2001].

5. Notons à ce propos que, d'une part, une analyse de sensibilité par rapport au taux d'intérêt sera conduite, et, d'autre part, que l'évaluation quantitative sera également effectuée plus spécifiquement pour le taux d'intérêt d'équilibre.

6. Cette valeur arbitraire n'a aucune incidence sur notre évaluation du coût des risques associés au marché du travail en présence d'épargne de précaution. Elle permet uniquement de déterminer le coût des fluctuations de revenu dans une économie sans aucune possibilité d'assurance. 
Il reste à étalonner le niveau des allocations-chômage qui n'existe que dans le cas de l'économie $A C$ : nous considérons un ratio de remplacement de $60 \%$ (MARTIN [1996]).

\subsection{Méthodes de résolution numérique de l'économie avec accumulation}

Les ménages accumulent un actif $k_{t} \in \mathcal{K}$, où $\mathcal{K}$ est une grille de $n$ valeurs $\left[0<k_{1}<k_{2}<\ldots<k_{n}\right]$ dans laquelle l'agent est contraint de choisir son épargne (contrainte d'endettement implicite car $k_{t} \geqslant 0 \forall t$ ).

Etant donnés $(w, r)$, vérifiant $\beta(1+r)<1$, et des valeurs données de $\left(k_{0}, s_{0}\right)$, l'agent doit choisir la séquence optimale de $\left\{k_{t}\right\}_{t=1}^{\infty}$ qui maximise la somme actualisée de ces flux d'utilité, sous ses contraintes budgétaires, de non-endettement et de positivité de sa consommation. Pour chaque $i \in[1, \ldots, n]$ (les points de la grille de capital), l'équation de Bellman de ce programme est :

$$
\begin{aligned}
& v\left(k_{i}, e\right)=\max _{k^{\prime} \in \mathcal{K}}\left\{u\left((1+r) k_{i}+w-k^{\prime}\right)+\beta\left[\pi_{e e} v\left(k^{\prime}, e\right)+\pi_{e u} v\left(k^{\prime}, u\right)\right]\right\} \\
& v\left(k_{i}, u\right)=\max _{k^{\prime} \in \mathcal{K}}\left\{u\left((1+r) k_{i}+\theta w-k^{\prime}\right)+\beta\left[\pi_{u e} v\left(k^{\prime}, e\right)+\pi_{u u} v\left(k^{\prime}, u\right)\right]\right\}
\end{aligned}
$$

On définit deux vecteurs $v_{j}$ de dimension $n \times 1$, pour $j=e, u$, où les lignes sont du type $v_{j}(i)=v\left(k_{i}, s_{j}\right)$ pour $i=1, \ldots, n$ et $j=e, u$. Soient deux matrices $R_{j}$ de taille $n \times n$ telles que :

$$
R_{j}(i, h)=u\left((1+r) k_{i}+w v\left(s_{j}\right)-k_{h}\right)
$$

pour $i=1, \ldots, n ; h=1, \ldots, n$ et $j=e, u$

On définit alors un opérateur $T\left(\left[v_{e}, v_{u}\right]\right)$ qui associe le couple de vecteur $\left[v_{e}, v_{u}\right]$ à un nouveau couple $\left[t v_{e}, t v_{u}\right]$. Cette application vérifie :

$$
\begin{aligned}
& t v_{e}=\max \left\{R_{e}+\beta \pi_{e e} \mathbf{1} v_{e}^{\prime}+\beta \pi_{e u} \mathbf{1} v_{u}^{\prime}\right\} \\
& t v_{u}=\max \left\{R_{u}+\beta \pi_{u e} \mathbf{1} v_{e}^{\prime}+\beta \pi_{u u} \mathbf{1} v_{u}^{\prime}\right\}
\end{aligned}
$$

où 1 est un vecteur $n \times 1$ contenant uniquement des 1 . On peut réécrire, sous forme matricielle, l'expression précédente :

$$
\left[\begin{array}{l}
t v_{e} \\
t v_{u}
\end{array}\right]=\max \left\{\left[\begin{array}{l}
R_{e} \\
R_{u}
\end{array}\right]+\beta(\pi \otimes \mathbf{1})\left[\begin{array}{l}
v_{e}^{\prime} \\
v_{u}^{\prime}
\end{array}\right]\right\}
$$

L'équation de BELLMAN peut alors s'écrire :

$$
\left[v_{e}, v_{u}\right]=T\left(\left[v_{e}, v_{u}\right]\right)
$$

et peut être résolue en itérant, jusqu'à convergence de la suite suivante :

$$
\left[v_{e}, v_{u}\right]_{m+1}=T\left(\left[v_{e}, v_{u}\right]_{m}\right)
$$


Les fonctions-valeur permettent de déterminer une application entre un niveau d'actif quelconque $i \in[1, n]$, et le numéro de la grille, i.e. $h \in[1, n]$, correspondant au choix optimal d'épargne $k^{\prime}$. Cette application permet de définir les règles de décisions individuelles $k^{\prime}=g(k, s)$. Numériquement l'ensemble des valeurs admissibles pour l'actif financier appartient à l'intervalle $[0,15]$. Le pas entre chaque point de cette grille est fixé à 0.0075 ce qui implique $n=2000$ valeurs possibles. Afin d'affiner ces résultats nous procédons ensuite à un lissage polynomiale des règles de décision (utilisation des polynômes de CHEBYSHEv) donnant alors une approximation des règles de décision sur un nouveau support entre $[0,15]$ où $n=10000$ points.

Une fois obtenues les règles de décisions individuelles $k^{\prime}=g(k, s)$, il est alors possible de calculer la distribution stationnaire de la richesse de cette économie. Cette distribution stationnaire est définie sur l'ensemble des paires $\left(k_{t}, s_{t}\right)$ réalisable et est résumée par :

$$
\lambda_{t}(k, s)=\operatorname{Prob}\left(k_{t}=k, s_{t}=s\right)
$$

sachant que la loi d'évolution de cette distribution est donnée par

$$
\begin{array}{r}
\operatorname{Prob}\left(k_{t+1}=k^{\prime}, s_{t+1}=s^{\prime}\right)=\sum_{s_{t}} \sum_{k_{t}} \operatorname{Prob}\left(k_{t+1}=k^{\prime} \mid k_{t}=k, s_{t}=s\right) \\
\operatorname{Prob}\left(s_{t+1}=s^{\prime} \mid s_{t}=s\right) \operatorname{Prob}\left(k_{t}=k, s_{t}=s\right)
\end{array}
$$

Etant donnée notre définition de $\lambda_{t}(k, s)$, elle peut encore s'écrire :

$$
\lambda_{t+1}\left(k^{\prime}, s^{\prime}\right)=\sum_{s} \sum_{k} \lambda_{t}(k, s) \operatorname{Prob}\left(s_{t+1}=s^{\prime} \mid s_{t}=s\right) \mathcal{I}\left(k, s, k^{\prime}\right)
$$

où $\mathcal{I}\left(k, s, k^{\prime}\right)$ est une variable indicatrice prenant la valeur un quand $k^{\prime}=g(k, s)$ et zéro sinon.

Afin de calculer la distribution stationnaire, on utilise le fait que l'économie est « markovienne » : chaque position se résume par le couple $(k, s)$ et évoluera en un couple $\left(k^{\prime}, s^{\prime}\right)$. On peut alors créer la « chaîne de MARKOV » décrivant l'évolution de l'économie à l'équilibre. Soit $\lambda$, tel que $\operatorname{dim}(\lambda)=2 n \times 1$, défini par :

$$
\lambda^{\prime}=\left[\lambda\left(k_{1}, e\right), \ldots, \lambda\left(k_{n}, e\right), \lambda\left(k_{1}, u\right), \ldots, \lambda\left(k_{n}, u\right)\right]
$$

La variable indicatrice $\mathcal{I}\left(k, s, k^{\prime}\right)$, de taille $n \times n$, indique le niveau de $k^{\prime}$, sachant que l'on avait $(k, s)$ donné par la règle de décision optimale $k^{\prime}=g(k, s)$. On peut alors écrire, avec $s \in\{e, u\}$ :

$$
\lambda_{t+1}^{\prime}=\lambda_{t}^{\prime}\left[\begin{array}{ll}
\pi_{e e} \mathcal{I}\left(k, e, k^{\prime}\right) & \pi_{e u} \mathcal{I}\left(k, e, k^{\prime}\right) \\
\pi_{u e} \mathcal{I}\left(k, u, k^{\prime}\right) & \pi_{u u} \mathcal{I}\left(k, u, k^{\prime}\right)
\end{array}\right]
$$

Une distribution est stationnaire si $\lambda_{t+1}=\lambda_{t}$, d'où :

$$
\lambda^{\prime}=\lambda^{\prime} \Pi \Longleftrightarrow\left(I-\Pi^{\prime}\right) \lambda=0
$$


où $\lambda$ est alors le vecteur propre associé à la valeur propre 1 de la matrice $\Pi$. On a alors, si $\Pi$ n'a qu'une seule valeur propre égale à un, une unique distribution stationnaire vérifiant :

$$
\lim _{t \rightarrow \infty} \lambda_{t}=\lambda
$$

Cette distribution stationnaire $\lambda$, donnant la probabilité inconditionnelle d'avoir le niveau d'actif $k$ et d'être dans l'état $s$ sur le marché du travail, peut également s'interpréter comme la fraction de temps pendant laquelle un individu conserve son état sur le marché du travail et dispose d'un certain montant d'actifs.

\subsection{Analyse des règles de décision}

La méthode qui vient d'être présentée permet de dériver les règles de décision et la distribution de la richesse pour les valeurs d'étalonnage préalablement spécifiées. Le graphique 1 représente la décision d'accumulation optimale $k^{\prime}$ étant donné le niveau précédent d'actifs $k$. On remarque que le travailleur épargne (pour un motif de précaution) pour des valeurs d'actif inférieures à 13,5 (la règle de décision coupe la droite à 45 degrés en ce point) ${ }^{7}$. En revanche, les chômeurs désépargnent quel que soit leur niveau d'actif (la règle de décision est toujours inférieure à la droite à 45 degrés). On remarque ${ }^{8}$ que, pour des niveaux faibles d'actifs, la contrainte de non-endettement est saturée.

\section{GRAPHIQUE 1}

\section{Règles de décision d'accumulation}
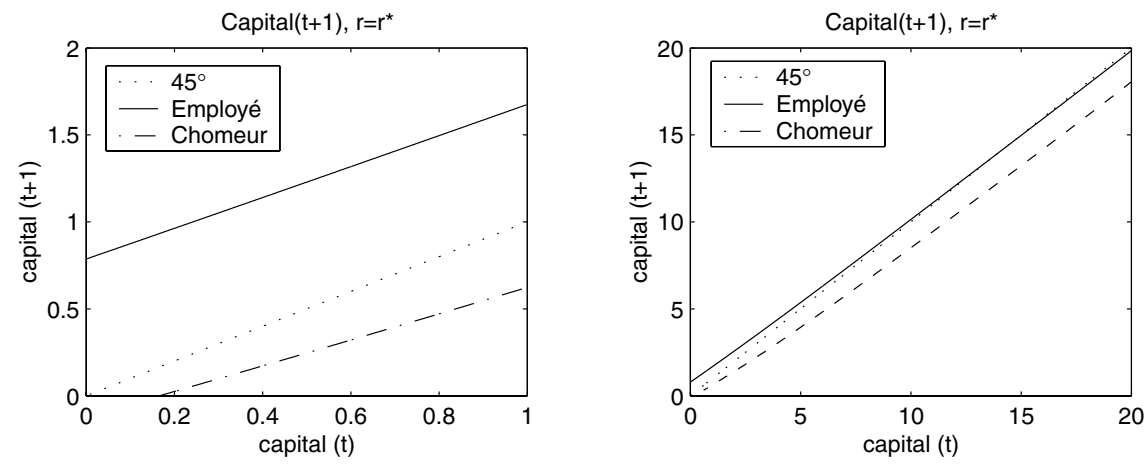

7. Sur le graphique de droite, on vérifie ex-post que ce point d'intersection est plus petit que la valeur maximale admissible pour le stock d'actif : l'existence de cet ensemble ergodique ainsi déterminé de façon endogène est une condition suffisante pour assurer l'existence et l'unicité de la distribution stationnaire de la richesse. Notons que lors des déviations du cadre de référence envisagées par la suite, la grille sera ajustée en conséquence.

8. Le graphique de gauche se focalise sur de faibles niveaux d'actifs. 
Ces décisions optimales d'accumulation impliquent des niveaux de consommation représentés sur le graphique 2 . On remarque alors un « coude » dans la règle de consommation des chômeurs correspondant aux deux régimes qu'ils peuvent connaître : (i) saturer la contrainte de liquidités et consommer le revenu courant (première partie linéaire de la règle de décision), (ii) puis, pour des niveaux d'actif plus élevés, avoir un comportement de lissage intertemporel de sa consommation permis par la détention d'actifs accumulés lorsqu'ils étaient employés.

GRAPHIQUE 2

Règles de décision de consommation
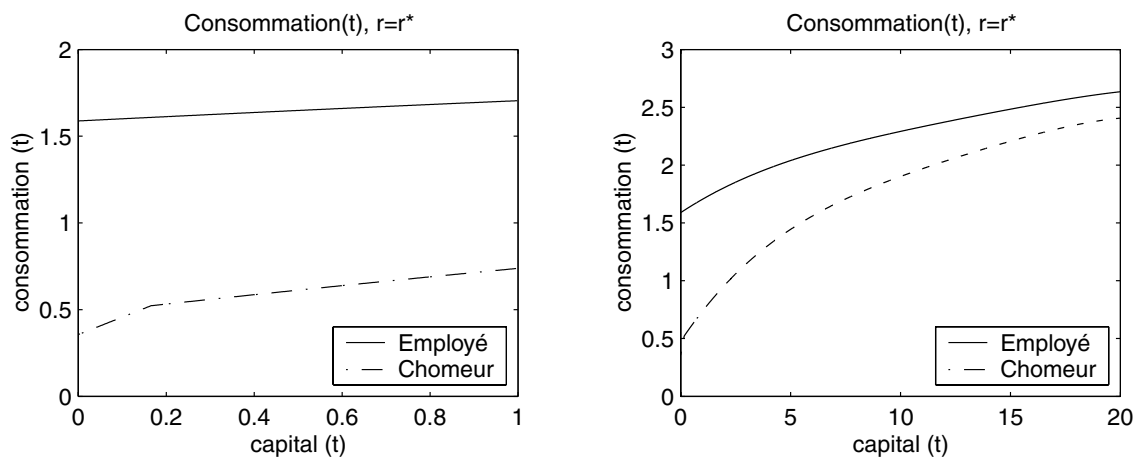

\section{5 Épargne et risque de chômage : une évalution quantitative}

L'objectif de cette section est d'évaluer l'épargne de précaution par rapport à l'assurance chômage en tant que mécanisme d'assurance au regard d'un système de mutualisation complète des risques.

\subsection{Mesure du coût associé au risque de chômage}

A combien les ménages vivant dans une économie avec assurance complète doivent-ils renoncer de consommation permanente pour supporter des systèmes assurantiels incomplets ? A la suite de LuCAS [1987], nous déterminons la fraction constante de consommation dont seraient privés les ménages s'ils devaient passer d'une économie où l'assurance complète est possible à des économies où seuls des systèmes incomplets d'assurance sont à leur disposition (marchés financiers, allocation-chômage ou aucune forme d'assurance). Cette fraction de consommation perdue est calculée comme le 
pourcentage $\mu$ de consommation permanente $(C)$ d'une économie avec une assurance complète qu'il faudrait retirer aux agents vivant dans cette économie pour qu'ils soient indifférents entre l'assurance complète et l'assurance incomplète. $\mu$ est tel que le niveau de bien-être atteint par un système de marchés complets coïncide avec celui, $U$, d'une économie avec assurance incomplète :

$$
U=\frac{1}{1-\beta} \frac{(C(1-\mu))^{1-\sigma}-1}{1-\sigma}
$$

\subsection{Auto-assurance versus allocation-chômage}

Dans le tableau 2, nous reportons le coût du risque de chômage associé respectivement à une économie avec une allocation-chômage et sans marchés financiers (notée $A C$ ), une économie avec marchés financiers mais sans allocation-chômage (notée $M F$ ) et une économie ne possédant aucun des deux mécanismes d'assurance (notée 0). Les pourcentages de consommation auxquels il faut renoncer sont respectivement notés $\mu_{M C \rightarrow A C}, \mu_{M C \rightarrow M F}$ et $\mu_{M C \rightarrow 0}$.

TABLEAU 2

Évaluation du rôle de l'épargne de précaution

\begin{tabular}{|ccc|}
\hline$\mu_{M C \rightarrow A C}$ & $\mu_{M C \rightarrow M F}$ & $\mu_{M C \rightarrow 0}$ \\
\hline 0,43 & 0,62 & 30,27 \\
\hline
\end{tabular}

Pertes de consommation en pourcentage de la consommation permanente dans une économie où l'assurance est complète $(C)$.

Il apparaît qu'une allocation-chômage dont le ratio de remplacement correspond à celui existant en France laisse persister des risques de revenu dont le coût est faible : il ne serait nécessaire de réduire la consommation de l'individu moyen vivant dans une économie avec assurance optimale que de $0,43 \%$, pour que celui-ci soit indifférent entre sa situation et celle qu'il atteindrait si l'assurance chômage était proche de celle observée en France. Permettre aux agents de s'auto-assurer via la constitution d'une épargne de précaution ne conduit pas les agents à amortir autant les variations de revenu dues au chômage. En effet, leurs décisions optimales d'épargne laissent persister des fluctuations de leur consommation dont le coût s'élève à $0,62 \%$. Toutefois, le coût du risque de chômage dans ces deux cas est considérablement réduit au regard d'une économie où les ménages prennent ces fluctuations de revenu de plein fouet. Dans cette dernière situation, il atteint en effet $30,27 \%$.

Ces résultats montrent que la possibilité de se constituer une épargne de précaution aboutit à des résultats relativement proches du système d'allocation-chômage, pour un ratio de remplacement réaliste. L'épargne de précaution permet en effet une couverture des risques relativement comparable au système d'allocation-chômage. Ainsi, un ménage vivant dans une 
économie où le risque de chômage est mutualisé via le système d'allocation chômage ne verrait sa consommation permanente baisser que de 0,19\% s'il ne pouvait plus que s'auto-assurer. Cette valeur représente le coût $\mu_{A C \rightarrow M F}$ qu'il y a à vivre dans une économie avec uniquement un marché financier par rapport à une autre où existe uniquement un système d'allocation-chômage.

\subsection{Auto-assurance et allocation-chômage}

Ces évaluations quantitatives sont construites sur la base de situations hypothétiques. Dans la réalité, il existe évidemment à la fois des possibilités d'épargne et une assurance publique. Le fait que l'auto-assurance ne permette pas d'atteindre l'assurance parfaite donne ainsi une légitimité à l'assurance publique. Si l'on couple auto-assurance et assurance publique, on diminue le coût associé au chômage de $\mu_{M C \rightarrow A C+M F}=0,29 \%$.

De façon générale, l'évaluation des gains de l'auto-assurance et de l'allocation-chômage sont indissociables. Le montant de l'épargne de précaution dépend des risques sur le marché du travail qui dépendent de l'allocationchômage, tandis que les coûts associés à une baisse de l'allocation-chômage dépendent de la possibilité de s'auto-assurer. Le gain lié à l'introduction de l'assurance-chômage dépend de la possibilité ou non de s'auto-assurer. Ceci peut se mesurer en comparant les gains de l'introduction de l'assurance chômage dans une économie sans marchés financiers à ceux où il existe des marchés financiers. Dans une économie sans marchés financiers, l'introduction de l'assurance chômage réduit le coût des fluctuations de revenu de $\left(\mu_{M C \rightarrow 0}\right)-\left(\mu_{M C \rightarrow A C}\right)=29,84$ points, tandis qu'il n'est diminué que de $\left(\mu_{M C \rightarrow M F}\right)-\left(\mu_{M C \rightarrow A C+M F}\right)=0,33$ point lorsque l'on peut préalablement s'auto-assurer.

Que représente le montant moyen de l'épargne de précaution $(K)$ ? Pour l'étalonnage de référence, il représente 4,11 fois le niveau du salaire. La provision optimale que constitue cette épargne de précaution est donc de l'ordre d'une année de salaire. Evidemment, ce montant dépend du risque associé au marché du travail et décroît avec la générosité de l'allocation-chômage (voir le tableau 3). Pour un risque correspondant à l'allocation-chômage effectivement versée, on constate que l'épargne de précaution correspond à un niveau négligeable d'épargne. La faiblesse de cette dernière ne constitue toutefois pas la preuve que les agents ne souhaitent pas utiliser dans l'absolu cette forme d'auto-assurance. Il s'agit du montant d'épargne optimal pour les ménages étant donné le niveau de l'allocation-chômage.

TABLEAU 3

\section{Montant de l'épargne de précaution et niveau de risque}

\begin{tabular}{|cccc|}
\hline$b$ & 0 & 0,30 & 0,60 \\
\hline$\frac{K}{w}$ & 4,11 & 1,29 & 0,07 \\
\hline
\end{tabular}




\subsection{Transferts intra-temporels et inter-personnels versus transferts inter-temporels et intra-temporels}

Une simulation permet de mieux se représenter les différences entre les systèmes d'assurance (voir la figure 3 ). Nous considérons un individu disposant d'un stock d'actifs égal à la moyenne de l'économie confronté à une histoire particulière sur le marché du travail correspondant à des réalisations de la variable aléatoire $s$ tirées au hasard selon le processus de MARKOV $\pi$. L'historique des transferts d'assurance permettant ces choix de consommation est décrit dans la figure 4. Celui-ci fait clairement appaître les réalisations des états sur le marché du travail : dans l'économie avec allocation-chômage, les transferts positifs sont associés au montant des cotisations salariales versées par un employé pour financer l'indemnisation des chômeurs $(\tau w)$. En période de chômage, le transfert correspondant au versement de l'indemnité est alors négatif. Ces transferts s'opèrent à l'intérieur de la période (intra-temporel) et entre les agents n'ayant pas le même état (inter-personnel).

Dans le cas de l'auto-assurance, les transferts sont inter-temporels mais intra-personnels. Ces transferts correspondent à l'effort d'épargne net de la rémunération des intérêts sur les placements passés $\left(k^{\prime}-k-r k\right)$. Les périodes d'emploi sont des épisodes où les ménages se constituent un matelas financier pour faire face aux épisodes futurs de chômage.

La dynamique de la consommation met en évidence une différence essentielle entre le système d'assurance publique et l'auto-assurance : la consommation dans chaque état est constante dans le premier, tandis qu'elle dépend de la position financière de l'agent dans le second. Dans le quatrième épisode de chômage, correspondant à la plus longue période de chômage, la désaccumulation est si forte que le consommateur se rapproche du niveau minimal de consommation, ce qui ne se produit jamais dans le cas de l'allocation-chômage. De façon générale, si la période de chômage est suffisamment longue, l'agent, même en puisant dans son épargne de précaution, est obligé de réduire sa consommation au-delà de ce qu'il aurait fait avec l'allocationchômage. Ce résultat illustre les travaux empiriques menés par (GRUBER [1994]) sur données américaines : celui-ci estime que la baisse de la consommation suite à une perte d'emploi est de seulement $6,8 \%$ avec le système d'allocation chômage, alors qu'elle serait de $22 \%$ en son absence. En revanche, si la période d'emploi est suffisamment longue, il peut bénéficier d'une consommation plus élevée. Sur la figure 3, ceci se produit par exemple lors des deux premiers épisodes d'emploi. De ce point de vue, la volatilité de la consommation est plus élevée (voir tableau 4). En revanche, on remarque que les variations d'une période sur l'autre sont beaucoup plus lisses avec épargne de précaution.

TABLEAU 4

Variance de la consommation et systèmes d'assurance

\begin{tabular}{|cccc|}
\hline$M C$ & $M F$ & $A C$ & 0 \\
\hline 0 & 0.11 & 0.028 & 0.61 \\
\hline
\end{tabular}


FIGURE 3

Historique des consommations

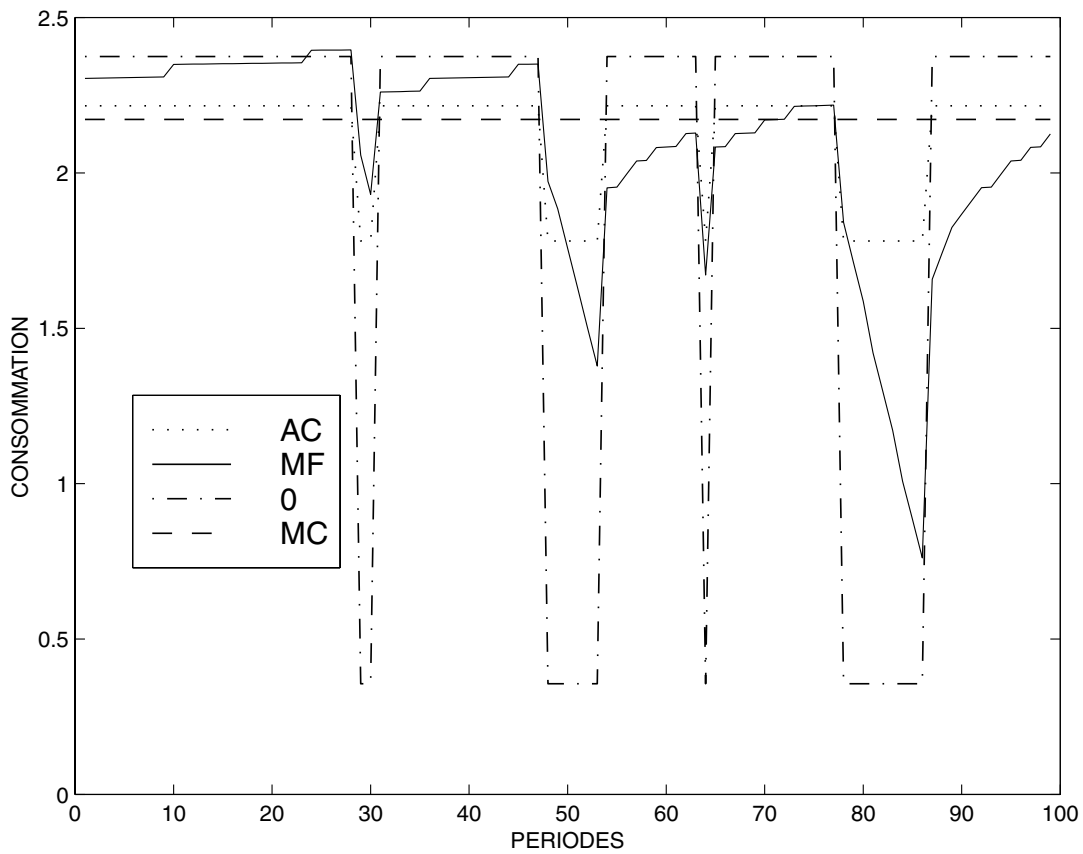

FIGURE 4

Historique des transferts

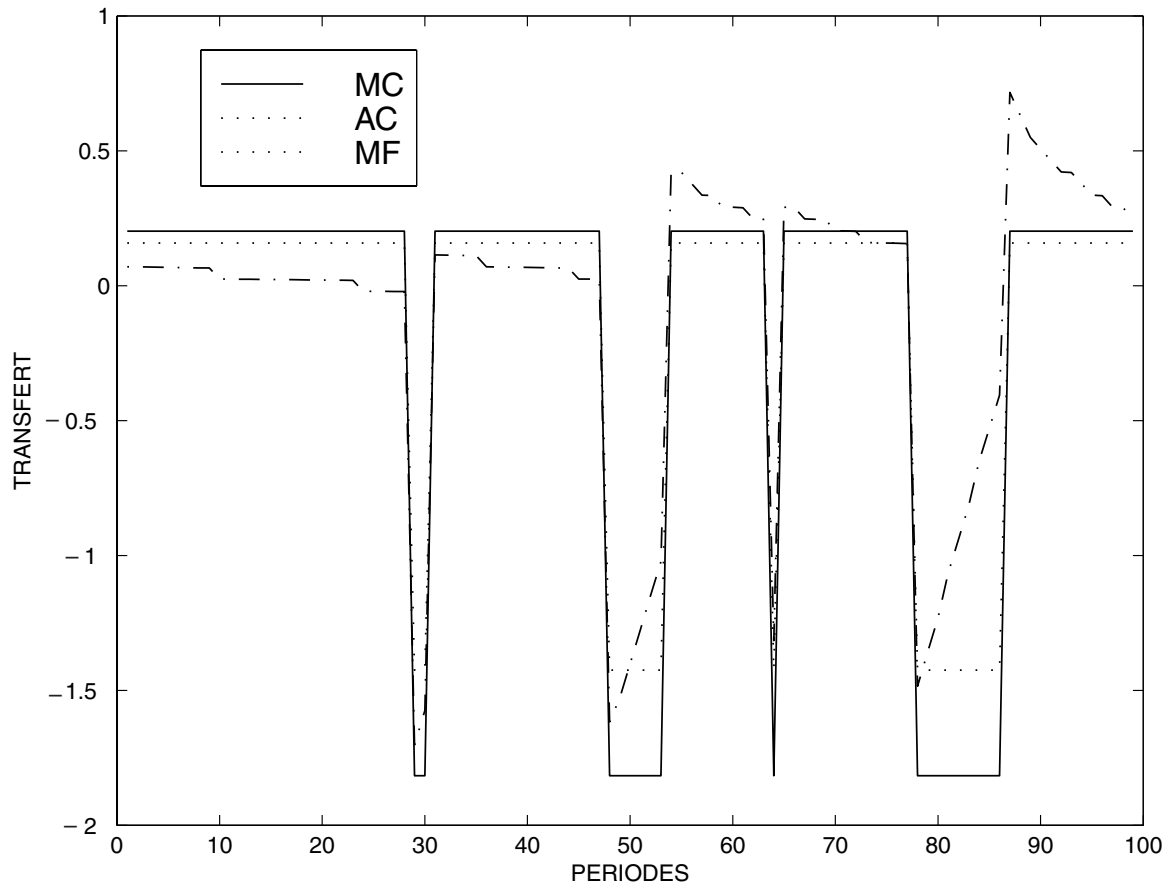

ÉPARGNE DE PRÉCAUTION ET CHÔMAGE 121 
Toutefois, le mécanisme d'assurance apparaît clairement dans tous les cas : on consomme moins que son revenu lorsque l'on travaille et plus en situation de chômage. Dans le cas d'une allocation-chômage, cela se traduit par des transferts entre travailleurs et chômeurs (transferts intra-temporels et interpersonnels). Dans le cas de l'auto-assurance, cela correspond à des transferts personnels dans le temps, entre périodes de travail et périodes de chômage. La décroissance de l'effort d'épargne reflète alors la baisse de l'incitation à placer de l'argent à mesure que le stock d'actif $(k)$ est grand. On constate que la désépargne (transferts reçus en période de chômage) ou l'épargne (transferts versés en période de travail) sont comparables aux transferts induits par le système d'allocation-chômage (figure 4).

\section{Les limites de l'épargne de précau- tion}

L'épargne de précaution est apparue dans la section précédente comme un moyen d'assurance alternatif à l'allocation-chômage. Ce résultat est d'autant plus intéressant que cette dernière induit des inefficacités ${ }^{9}$ qui, même si elles ne sont pas prises en compte dans ce travail, pourraient faire basculer les résultats en faveur de l'auto-assurance. Cependant, il existe de nombreuses limites à cette dernière qui n'apparaissent pas dans l'analyse précédente. La première vient du fait que seuls des états stationnaires ont été considérés. Or, il est évident que la constitution d'une épargne de précaution peut introduire des coûts, certes transitoires, mais potentiellement importants. Une deuxième s'explique par la dépendance des résultats de l'auto-assurance par rapport au niveau du taux d'intérêt qui détermine son prix. Enfin, l'auto-assurance tend à particulièrement pénaliser les individus qui restent durablement au chômage. En effet, ne reposant pas sur la mutualisation des risques individuels, elle dépend de l'histoire particulière des agents. Nous examinons à tour de rôle ces différentes limites.

\subsection{La transition}

A l'état stationnaire, des économies avec un système public ou avec de l'épargne de précaution seraient relativement équivalentes en termes de bienêtre. Cependant, la prise en compte de la dynamique de transition rend coûteuse l'auto-assurance. Partant d'une situation de parfaite mutualisation des risques dans laquelle les agents ne détiennent aucun actif financier, la constitution d'une épargne de précaution, suppose de renoncer dans un premier temps à de la consommation. Comme le montre le tableau 5, le coût des fluctuations de revenu est alors significativement augmenté dans une économie

9. Nous pensons ici par exemple au problème d'aléa-moral sur le comportement d'offre de travail, mais aussi aux effets distorsifs du taux de cotisation. 
avec marchés financiers lorsque l'on prend en compte cette transition. Ainsi, on constate que le coût des fluctuations de revenu atteint $9,6 \%$ au lieu de $0,73 \%$ lors d'une simple comparaison entre états stationnaires. Comme le coût des fluctuations de revenu avec allocation-chômage reste inchangé, la différence est maintenant beaucoup plus importante.

TABLEAU 5

Evaluation du rôle de l'épargne de précaution avec transition

\begin{tabular}{|cc|}
\hline$\mu_{M C \rightarrow M F}$ & $\mu_{M C \rightarrow A C}$ \\
\hline 9,94 & 0,43 \\
\hline
\end{tabular}

Pertes de consommation en pourcentage de la consommation permanente dans une économie où l'assurance est complète $(C)$.

\subsection{Le niveaux du taux d'intérêt}

Le taux d'intérêt, ou plus précisément son inverse, détermine le prix de l'auto-assurance. L'offre de capital est croissante dans le taux d'intérêt (figure 5).

FIGURE 5

Offre de capital

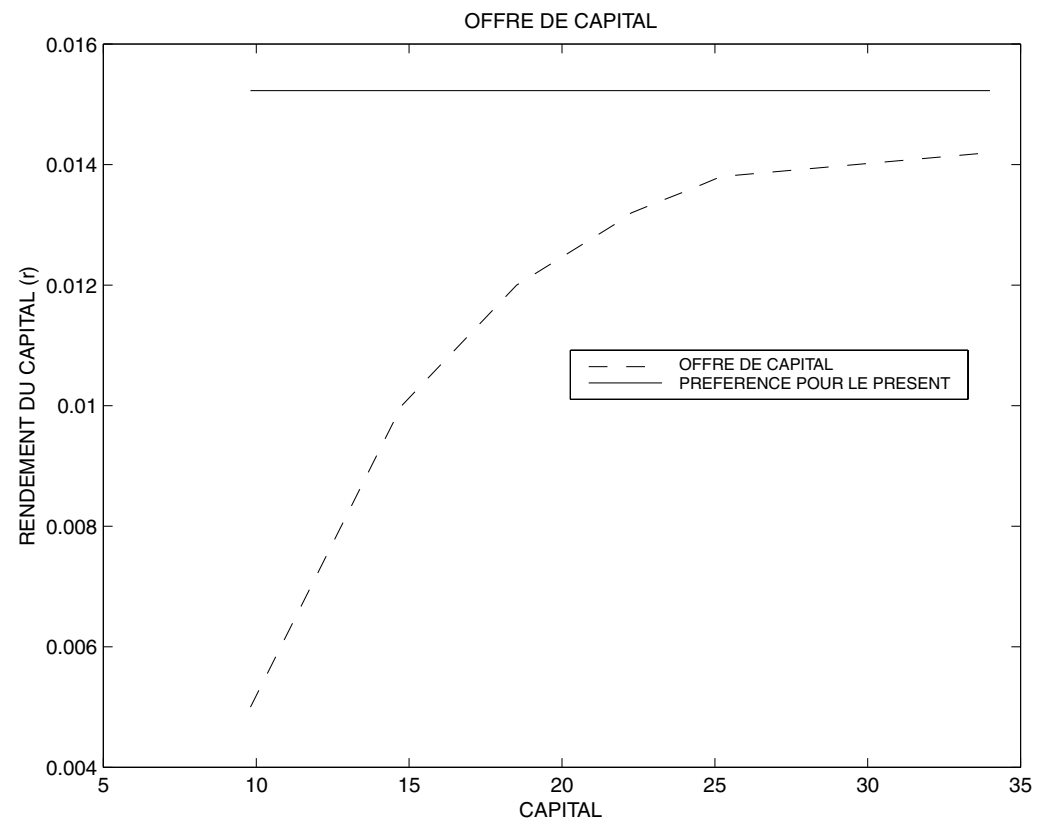


Les gains en bien-être induits par l'épargne de précaution croissent avec $r$ (tableau 6). De façon apparemment paradoxale, pour certains taux d'intérêt (pour $r=1 \%$ par exemple), on pourrait être mieux dans une économie risquée avec auto-assurance que dans une économie sans risque (avec assurance complète) si l'on ne considérait que l'état stationnaire. Ceci s'explique par un niveau de consommation moyen plus élevé obtenu grâce à des revenus d'intérêt plus importants, le coût transitoire de l'accumulation n'étant pas pris en compte. Il est donc particulièrement important de prendre en compte la transition.

\section{TABLEAU 6}

\section{Evaluation du rôle du taux d'intérêt}

\begin{tabular}{|ccccc|}
\hline$r$ & $K$ & $K / Y$ & $\begin{array}{c}\mu_{M C \rightarrow M F} \\
\text { (sans trans.) }\end{array}$ & $\begin{array}{c}\mu_{M C \rightarrow M F} \\
\text { (avec trans.) }\end{array}$ \\
\hline $0 \%$ & 8,73 & 3,72 & 3,73 & 11,35 \\
$0,5 \%$ & 9,91 & 3,95 & 0.62 & 9,94 \\
$1 \%$ & 12,7 & 5,1 & $-4,3$ & 8,25 \\
$1,38 \%\left(r^{\star}\right)$ & 21,3 & 8,17 & -14 & 6,67 \\
\hline
\end{tabular}

Pertes de consommation en pourcentage de la consommation permanente dans une économie où l'assurance est complète $(C)$.

La dépendance par rapport au taux d'intérêt plaide pour une analyse en équilibre général dans laquelle le taux d'intérêt résulte de la confrontation de l'offre et de la demande de capital. Comme dans le modèle d'AIYAGARI [1994], le taux d'intérêt d'équilibre est celui qui égalise l'offre de fonds des ménages et la demande des entreprises ${ }^{10}$. Le graphique 6 représente l'équilibre du marché du capital ${ }^{11}$. La valeur du taux d'intérêt d'équilibre est égale à 1,38\%. Les figures 7-9 en annexe retracent la distribution de la richesse entre les agents pour ce taux d'intérêt. Le coût des fluctuations de revenu est lui reporté dans le tableau 6. Ce taux d'intérêt d'équilibre, plus de 2,5 fois supérieur à celui considéré dans notre étalonnage de référence, permet de rendre moins coûteuse la transition, la rémunération des placements étant relativement forte ${ }^{12}$.

10. Voir HugGetT [1997] pour une modélisation d'un équilibre général sans production. Le taux d'intérêt permet alors d'équilibrer le marché financier où créanciers et débiteurs s'échange un actif. Notons que la modélisation proposée par HuGGETT [1997] nécessite donc une contrainte d'endettement permettant des positions débitrices, ce qui n'est pas envisagé dans notre étude.

11. Voir l'annexe B pour une présentation de la méthode de résolution de cet équilibre général avec production.

12. Pour $r=r^{*}$, l'évaluation du passage à une économie avec auto-assurance $\left(\mu_{M C \rightarrow M F}\right.$ avec transition.) est approximée par une trajectoire où le taux d'intérêt est supposé sauter instantanément sur sa valeur d'équilibre de long-terme. 


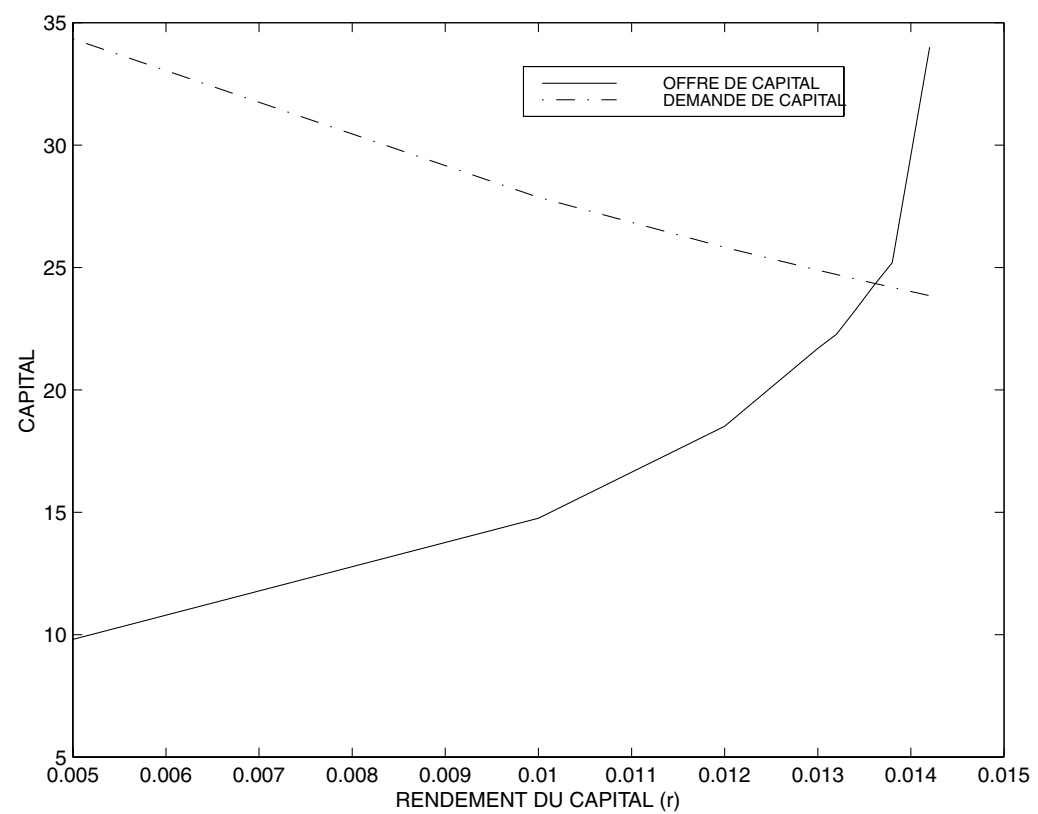

\subsection{Epargne de précaution et inégalités}

Nous choisissons dans un premier temps d'adopter un critère rawlsien consistant à se concentrer sur l'individu le plus défavorisé dans l'économie. Il s'agit d'un chômeur sans actif financier qui tire de façon perpétuelle le mauvais état de la nature, le chômage. Remarquons que l'on peut également interpréter cette situation comme l'état le plus défavorable à chaque période dans une économie stationnaire, sachant que cette position n'est pas occupée par le même individu. On peut alors parler d'inégalités de position. Le système d'auto-assurance coïncide dans ce cas avec l'économie sans possibilité d'assurance. En revanche, le système d'allocation-chômage permet de mieux assurer ce type de situation (Tableau 7). Si l'on compare avec les résultats reportés dans le tableau $1^{13}$, le coût du risque de chômage lorsque l'on ne prend en compte que le bien-être de cet agent défavorisé apparaît particulièrement augmenté en situation d'auto-assurance. De façon générale, un système d'allocation-chômage permet de s'assurer d'un minimum de revenu, même dans les circonstances les plus défavorables, tandis que l'auto-assurance dépend du stock d'actifs financiers disponible. Des périodes particulièrement prolongées de chômage ne peuvent pas être financées. Ceci est inhérent à l'épargne de précaution.

13. Dans le cas étudié, prendre en compte la transition n'a pas de sens, puisque l'agent concerné n'a pas d'actifs. 
Epargne de précaution et Inégalités de position

\begin{tabular}{|cc|}
\hline$\mu_{M C \rightarrow M F}$ & $\mu_{M C \rightarrow A C}$ \\
\hline 83,6 & 18,02 \\
\hline
\end{tabular}

Pertes de consommation en pourcentage de la consommation permanente dans une économie où l'assurance est complète $(C)$. Ces évaluations sont faites pour $r=0,5 \%$.

Cette vision des inégalités est cependant très particulière dans le sens où elle se polarise uniquement sur l'histoire d'un individu de masse nulle dans l'économie. Son niveau de bien-être ne correspond pas à son espérance de gains, puisqu'un chômeur a une probabilité non nulle de revenir sur le marché du travail. Cependant, cela n'implique pas que le raisonnement en espérance élimine les inégalités entre un chômeur et un travailleur. Au fur et à mesure que le temps s'écoule, les histoires sur le marché du travail tendent à se rejoindre en espérance, mais les conditions initiales en revanche influent sur les espérances de bien-être. A un moment donné dans l'économie, il existe une hétérogénéité en termes de situation sur le marché du travail et de détention d'actifs financiers qui implique des espérances de bien-être inégales. On peut alors parler d'inégalités de perspectives. Pour prendre en compte dans la mesure du bien-être collectif cette dimension spécifique aux inégalités, considérons la fonction de bien-être suivante :

$$
\mathcal{W}=\left(\int_{\mathcal{K}} \lambda_{t}(k, s) v_{t}(k, s)^{1-\xi} d k\right)^{\frac{1}{1-\xi}}
$$

où $\xi$ est un indicateur d'aversion aux inégalités. Plus il est élevé, plus la société se préoccupe des agents les moins favorisés (en termes d'espérance de bien-être). Remarquons que le critère rawlsien considéré précédemment consiste à considérer le cas où $\xi$ tend vers l'infini, mais dans un contexte où la probabilité du chômage tend vers zéro.

Les résultats reportés dans le tableau 8 peuvent apparaître assez surprenants. Soulignons d'abord qu'ils prennent en compte les coûts liés à la transition. Considérer un critère pénalisant les inégalités semble affecter particulièrement le système d'allocation-chômage. On constate que lorsque l'on prend en compte une aversion pour l'inégalité $(\xi>0)$, le coût des fluctuations de revenu augmente plus sensiblement dans ce cas, même si l'effet du coût de la transition rend encore beaucoup moins performante l'auto-assurance. Raisonner en termes d'espérance conduit en effet à mettre en évidence une autre dimension de l'auto-assurance. C'est dans la période d'activité que se produit l'effort d'épargne et donc une renonciation à la consommation supérieure à celle induite par le prélèvement fiscal finançant les allocationschômage. Cette situation était déjà apparente dans la figure 3 où la consommation en auto-assurance était beaucoup plus lisse d'une période sur l'autre : au moment du passage de l'état de chômeur à celui de travailleur, la 
Epargne de précaution et inégalités de perspectives

\begin{tabular}{|ccc|}
\hline$\xi$ & $\mu_{M C \rightarrow M F}$ & $\mu_{M C \rightarrow A C}$ \\
\hline 0 & 9,94 & 0,43 \\
2 & 10,05 & 0,95 \\
5 & 10,93 & 1,97 \\
\hline
\end{tabular}

Pertes de consommation en pourcentage de la consommation permanente dans une économie où l'assurance est complète $(C)$. Ces évaluations sont faites pour $r=0,5 \%$ et en prenant en compte le coût de la transition.

consommation augmente beaucoup plus avec allocation-chômage. L'autoassurance tend à réduire le différentiel de bien-être espéré entre chômeur et travailleur. Ce résultat n'est pas contradictoire avec celui obtenu avec un critère rawlsien car dans ce dernier cas on raisonne sur une situation qui ne correspond pas à l'espérance de bien-être d'un chômeur, mais sur l'histoire très particulière d'un individu.

Ainsi, la situation d'auto-assurance tend à défavoriser les individus subissant une période de chômage supérieure à la durée anticipée, tandis qu'elle semble créer moins d'inégalités en moyenne (en espérance) entre un chômeur et un travailleur. L'auto-assurance est ainsi plus inégalitaire en termes de position, mais moins en termes de perspectives.

\section{Conclusion}

Les agents ont la possibilité de constituer une épargne (de précaution) pour faire face au risque de chômage. Dans une économie stationnaire, cette autoassurance permet d'atteindre des niveaux de bien-être comparables à ceux garantis par le système public d'assurance-chômage. Ces résultats plaident pour prendre en compte l'accumulation d'actifs financiers dans les travaux sur l'allocation-chômage. Nous avons ainsi montré que l'évaluation des gains de l'auto-assurance et de l'allocation-chômage sont indissociables. L'auto-assurance dépend des risques sur le marché du travail qui dépendent de l'allocation-chômage, tandis que les coûts associés à une baisse de l'allocation-chômage dépendent de la possibilité de s'auto-assurer.

Toutefois, notre conclusion ne revient pas à dire que les marchés financiers sont suffisants pour assurer de façon optimale les ménages. D'abord, toute diminution du niveau de l'allocation-chômage entraîne un coût aujourd'hui imputable à l'effort d'accumulation supplémentaire pour se constituer dans la dynamique de transition une épargne de précaution plus élevée. Ce passage d'une économie stationnaire à une autre vient limiter l'attrait de l'auto-assurance. En outre, un système d'auto-assurance vient considérablement accroître les inégalités de position dans l'économie. L'hétérogénéité des trajectoires 
d'accumulation imputable aux histoires particulières sur le marché du travail rend la situation d'auto-assurance relativement plus inégalitaire pour les individus qui restent au chômage bien au-delà de la durée espérée. Le mérite de l'allocation-chômage est alors de maintenir le bien-être de ceux que l'on peut considérer comme les plus défavorisés en termes de réalisation des aléas sur le marché du travail. Cependant, en termes d'inégalités de perspectives, l'autoassurance semble relativement moins inégalitaire dans la mesure où elle repose sur des choix individuels intertemporels qui tendent à minorer l'écart de bien-être espéré entre un chômeur et un travailleur.

Naturellement, ces résultats ont été obtenus dans un cadre simplifié. Il serait utile de les approfondir dans le contexte d'une économie plus réaliste. Toutefois, ils permettent déjà de souligner tout l'intérêt qu'il y a prendre en compte les interdépendances entre les décisions d'accumulation et les comportements sur le marché du travail.

\section{A Graphiques des distributions}

FIGURE 7

\section{Distribution du capital pour le taux d'intérêt d'équilibre}

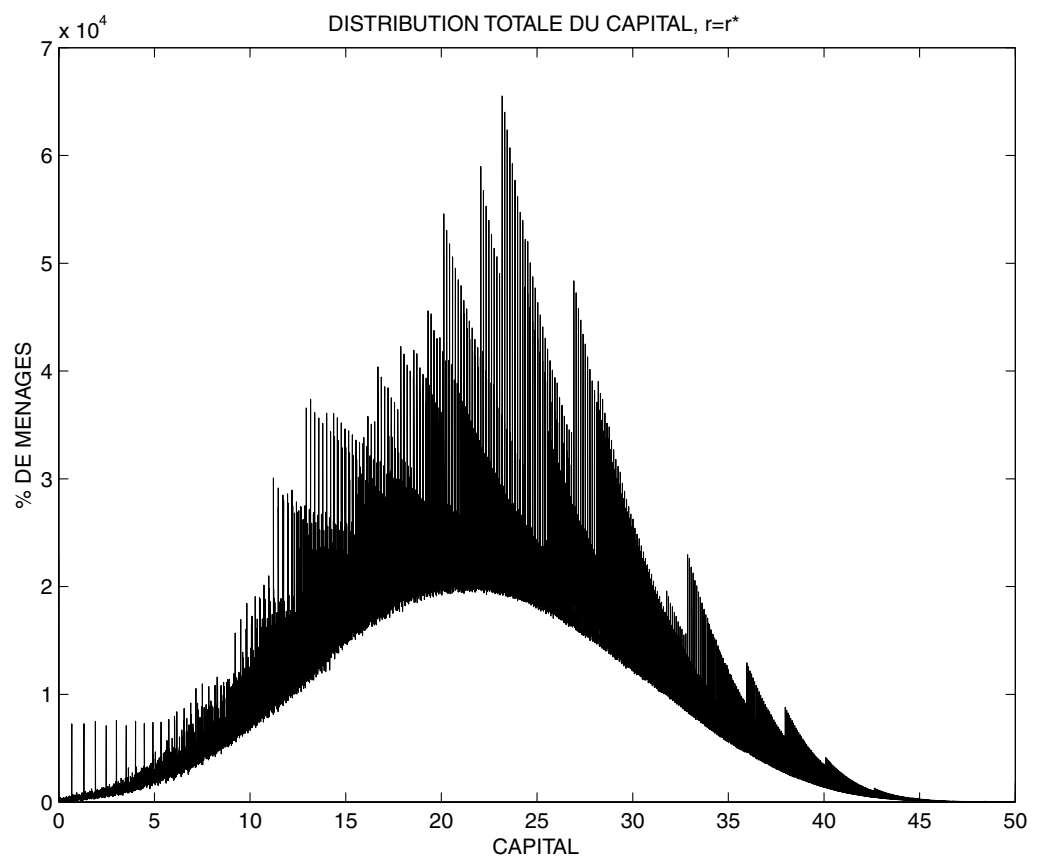


FIGURE 8

Distribution du capital (employés) pour le taux d'intérêt d'équilibre

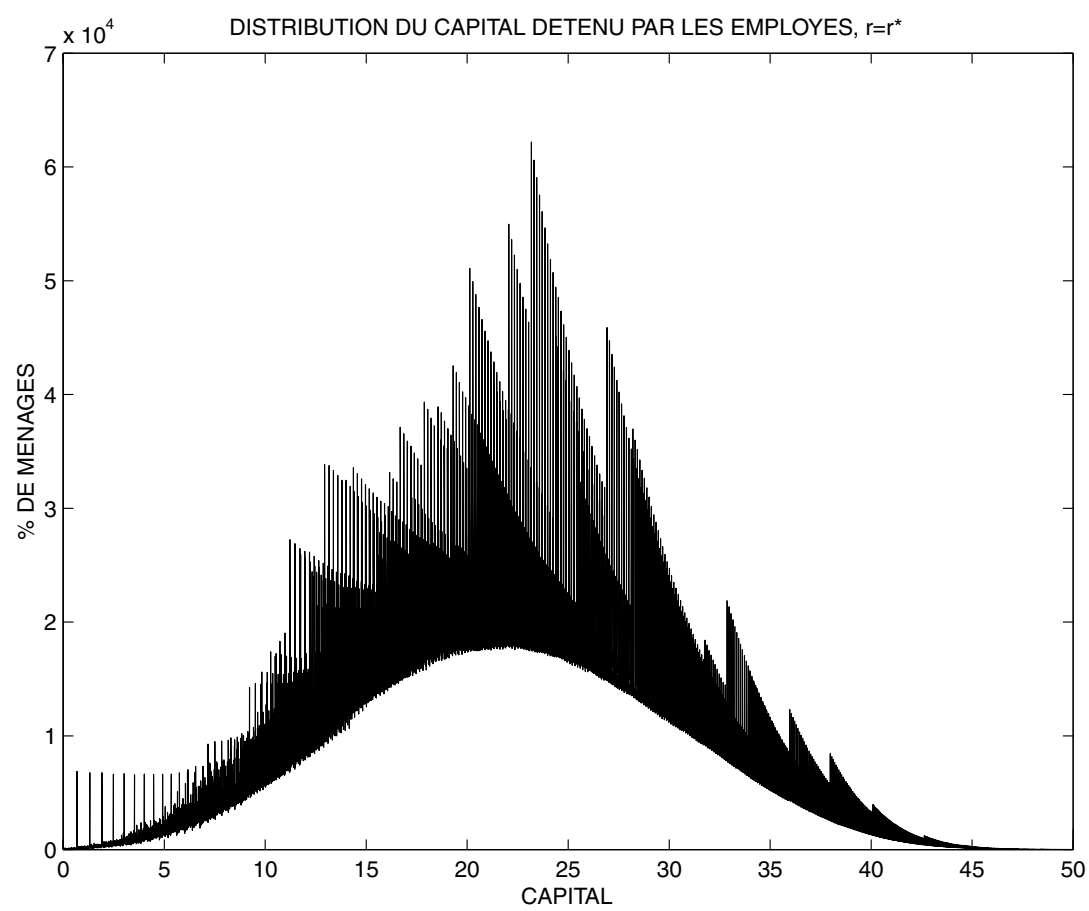

FIGURE 9

Distribution du capital (chômeurs) pour le taux d'intérêt d'équilibre

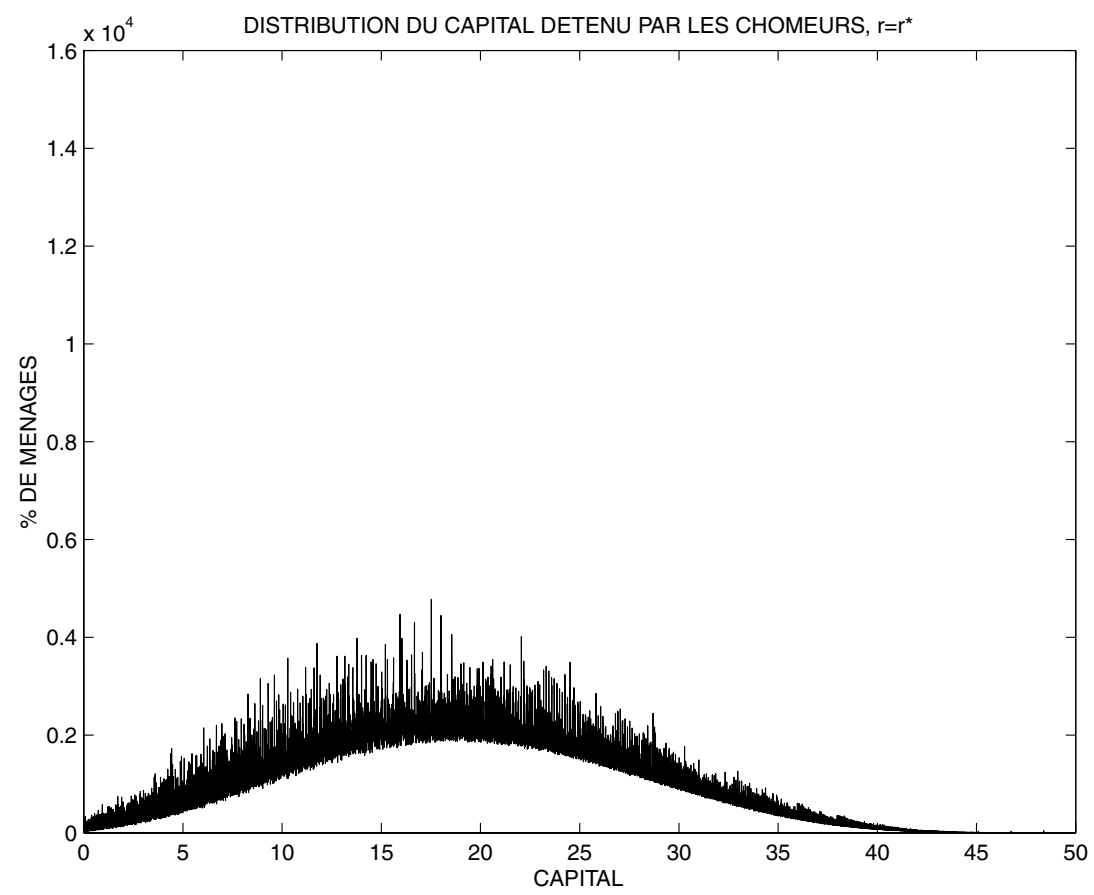




\section{B Equilibre général : méthode de réso- lution}

1. Pour une valeur donnée du stock de capital agrégé $K=K_{j}$, on calcule les prix des facteurs $\left(r_{j}, w_{j}\right)$ étant donnée la fonction de production $Y=A K^{\alpha} N^{1-\alpha}$ : on s'assure ainsi que les ajustements du taux d'intérêt nécessaire pour équilibrer le marché du capital s'accompagnent de modification du salaire le long d'une même $F P F$. On résout ensuite le problème des ménages. Etant données les règles de décision $k^{\prime}=g_{j}(k, s)$, on en déduit la distribution stationnaire $\lambda_{j}(k, s)$.

2. On calcule alors le stock de capital offert dans l'économie :

$$
K_{j}^{s}=\sum_{k, s} \lambda_{j}(k, s) g_{j}(k, s)
$$

3. Pour un paramètre $\xi$ donné, tel que $\xi \in(0,1)$, on calcule la nouvelle valeur de $K$ :

$$
K_{j+1}=\xi K_{j}+(1-\xi) K_{j}^{s}
$$

4. On répète cette boucle jusqu'à obtenir le point fixe sur le stock de capital $K^{*}$. On déduit alors $r^{*}=\alpha A K^{* \alpha-1} N^{1-\alpha}$. 